\title{
Adverse drug reactions to first line anti-tuberculosis drugs in newly diagnosed tuberculosis patients
}

\author{
Elakkya Kolanchinathan ${ }^{1 *}$, Dhanya Thirookaran Harichandran², \\ Sujatha Mangattuvalappil Balakrishnan ${ }^{1}$, Sanal Kumar Kondarappassery Balakumaran²
}

\author{
${ }^{1}$ Department of Pharmacology, Government Medical College, Thrissur, Kerala, India \\ ${ }^{2}$ Department of Pharmacology, Government Medical College, Manjeri, Kerala, India
}

Received: 16 November 2021

Accepted: 06 December 2021

\author{
*Correspondence: \\ Dr. Elakkya Kolanchinathan, \\ Email: elakkiyakr277@gmail.com
}

Copyright: (C) the author(s), publisher and licensee Medip Academy. This is an open-access article distributed under the terms of the Creative Commons Attribution Non-Commercial License, which permits unrestricted non-commercial use, distribution, and reproduction in any medium, provided the original work is properly cited.

\begin{abstract}
Tuberculosis (TB) continues to be an important public health problem throughout much of the world. Drug treatment is the only effective treatment method, but adverse drug events (ADEs) and adverse drug reactions (ADRs) can affect medical adherence. As the number of drug resistant TB patients and the number of anti-TB drugs have increased, it is necessary to explore the risk factors for ADEs/ADRs to reduce their occurrence. Here we reported three different cases of ADRs due to first line anti-tubercular drugs.
\end{abstract}

Keywords: Tuberculosis, Anti- tubercular drugs, Adverse drug events, Adverse drug reactions

\section{INTRODUCTION}

TB is an infective bacterial disease caused by Mycobacterium tuberculosis, which mainly affects the lungs. In 2017, approximately 10.0 million people developed TB disease and TB caused an estimated 1.3 million deaths. ${ }^{1}$ Drug treatment is the only effective method for TB and the WHO has implemented a standardised directly observed treatment, the short course (DOTS)/stop TB strategy, to improve TB prevention and control. Although the WHO set the global target rate for a successful treatment outcome at $85 \%$, the treatment success rate remains low at $82 \%$ for all TB patients and $55 \%$ for drug resistant TB (DR-TB) patients. ${ }^{1,2}$ Many factors have influenced the treatment success rate, among them patient's compliance to prescribed regimen and ADEs and ADR are significant factors affecting the adherence of patients to effective chemotherapy. ${ }^{3}$

Long term anti-TB combination regimens could lead to various types and levels of ADEs/ADRs which could subsequently lead to treatment discontinuation or interruption, treatment time extension and an increased risk for developing drug resistance, treatment failure and relapse. ${ }^{4}$ All of these negative consequences have posed a challenge to TB treatment and have implications for TB control.

Among the various ADEs/ADRs, the most common ones induced by first line anti-TB drugs are hepatotoxicity, gastrointestinal disorders, allergic reactions, arthralgia, neurological disorders, while in multidrug-resistant TB (MDR-TB) patients, common types are arthralgia, gastrointestinal disorders, hypothyroidism, dermatologic disorders, haematological disorders, hepatotoxicity, ototoxicity and nervous system or psychiatric disorders. ${ }^{5,6}$ TB patients treated by second-line anti-TB drugs in the context of high HIV coinfection had more severe ADEs/ADRs and the most common severe type reported was hearing loss or ototoxicity. ${ }^{7}$ As problems such as DRTB, MDR-TB, extensively drug-resistant TB (XDR-TB), and a recently emerging threat by a totally drug-resistant 
TB (TDR-TB) are increasing in prevalence, several novel drugs will cause a significant shift in the landscape of TB treatment but can cause some other ADEs/ADRs. For example, bedaquiline and delamanid can prolong cardiac QTc. ${ }^{8} 9$ Treatment interruption due to ADEs/ADRs could decrease the chance of a cure and lead to patient loss to follow up, further increasing the development of drug resistance. ${ }^{10,11}$ Therefore, it is necessary to strengthen research on ADEs/ADRs in anti-TB treatment.

Here, we reported one case of exfoliative dermatitis and two cases of drug induced liver injury (DILI) due to first line anti-TB drugs in our tertiary care hospital. Causality assessment and severity and preventability assessment was done.

\section{CASE SERIES}

\section{Case 1}

A 73 year old female patient, a diagnosed case of sputum positive pulmonary tuberculosis, on first line antitubercular treatment for nine weeks, who have completed intensive phase started on continuation phase. She presented to dermatology OPD with complaints of itching and generalised rash all over the body. She had a past history of hypertension for which she was on treatment (amlodipine $5 \mathrm{mg}$, telmisartan $40 \mathrm{mg}$ ) for 20 years. On examination, she was febrile and had facial puffiness, edema of dorsum of hands and feet and there was generalised scaling eruption involving the face, neck, trunk, extremities and palms. A diagnosis of exfoliative dermatitis due to antitubercular treatment was made. Antitubercular treatment was then withheld. She was started on corticosteroids and antihistaminics.

Further workup revealed a normal total leukocyte count (TLC-8000), normal liver and kidney function tests, ELISA for HIV negative and sequential chest radiography showed radiological improvement.

Once her rash completely resolved, drugs were reintroduced according to WHO recommendations (Figure 1). On reintroduction of rifampicin $(300 \mathrm{mg})$, she developed increase in itching and rash. Rifampicin was then withdrawn. But she tolerated isoniazid, ethambutol. She was suspected as a case of rifampicin induced exfoliative dermatitis. Modified anti tubercular therapy with isoniazid, ethambutol and streptomycin (HSE therapy) was started in continuation phase. She well tolerated to HSE therapy. Steroids were gradually tapered and stopped. After five months of (anti-tubercular treatment) ATT, patient showed clinical and radiological improvement.

\section{Case 2}

A 66 years old male patient, a diagnosed case of sputum positive pulmonary TB, on first line ATT for two weeks presented to pulmonology OPD with complaints of generalized weakness, loss of appetite, yellowish discolouration of eyes and altered behaviour. The patient had a past history of DM for 25 years was on treatment. On general examination, he was conscious, febrile with temperature of $99{ }^{\circ} \mathrm{F}$, pulse rate of 89 beats per minute, blood pressure of $160 / 100 \mathrm{~mm}$ of $\mathrm{Hg}$ and icteric. Respiratory examination revealed bilateral coarse crackles and wheezes. Per abdomen examination revealed soft and distended abdomen with palpable hepatomegaly.

On laboratory examination, prothrombin time-16.4 sec, INR-1.17, aPTT-49 sec. USG abdomen revealed moderate hepatomegaly with mild ascites. The liver function test parameters were markedly elevated, AST-382U/1, ALT360U/1, ALP-153U/1, total bilirubin was found to be 6.7 $\mathrm{mg} / \mathrm{dl}$. Hepatic serological panel of test were negative. Based on the triad of the rapid loss in hepatocyte function (jaundice, coagulopathy and ascites), absence of a prior history of liver disease, the diagnosis of ATT induced liver injury was established. All his medications were promptly discontinued and the supportive treatment was started with humified oxygen, N-acetyl cysteine $600 \mathrm{mg}$, heptogen, fresh frozen plasma (FFP) transfusion as needed, empiric antimicrobial coverage (cefaperazone+salbactum $1 \mathrm{~g}$ intravenously every 8 hour), nutritional support (a high protein and salted diet) and iron folic acid tablets. Childpug scoring was done with score of 7 . The lab parameters were improved after two weeks, patient condition improved considerably over a week. ATT drugs were reintroduced according to WHO recommendations. Patient tolerated rifampicin, isoniazid and ethambutol. When he was started on pyrazinamide $1000 \mathrm{mg}$, his liver parameters again increased. Hence the drug was suspected as pyrazinamide according to temporal relationship. Other hepatotoxic drugs like rifampicin and isoniazid were also stopped. Modified ATT therapy with streptomycin, levofloxacin, ethambutol (SLE therapy) were started. After two months of ATT, patient showed clinical improvement.

\section{Case 3}

A 74 year old male patient, a newly diagnosed case of sputum positive pulmonary tuberculosis, on first line antitubercular drugs for 10 days presented to pulmonology OPD with complaints of generalised weekness, loss of appetite. He was a known case of diabetes and hypertension for 30 years on treatment. On general examination, he was conscious, afebrile, pulse rate of 85 beats per minute, blood pressure of $170 / 90 \mathrm{~mm}$ of $\mathrm{Hg}$. Icterus and clubbing were present. Respiratory examination showed bilateral coarse crackles. Per abdomen examination revealed soft and distended abdomen.

On laboratory investigations, TLC was elevated (TC13500), RBS-212 mg/dl, serum Na+ 126 mg/dl, serum K+ $4.4 \mathrm{mg} / \mathrm{dl}$. The liver function parameters were elevated, total serum bilirubin was $2.6 \mathrm{mg} / \mathrm{dl}$, AST-100U/1, ALT-96 U/1, ALP-108 U/l. Chest radiography revealed right pleural effusion. USG abdomen revealed ascites. A diagnosis of 
drug induced liver injury was established. All ATT drugs were promptly stopped. He was started on rifagut $550 \mathrm{mg}$, $\mathrm{N}$-acetyl cysteine $600 \mathrm{mg}$, syrup lactulose. Pleural fluid aspiration was done under local anaesthesia and aseptic precautions. Pleural fluid examination was done.

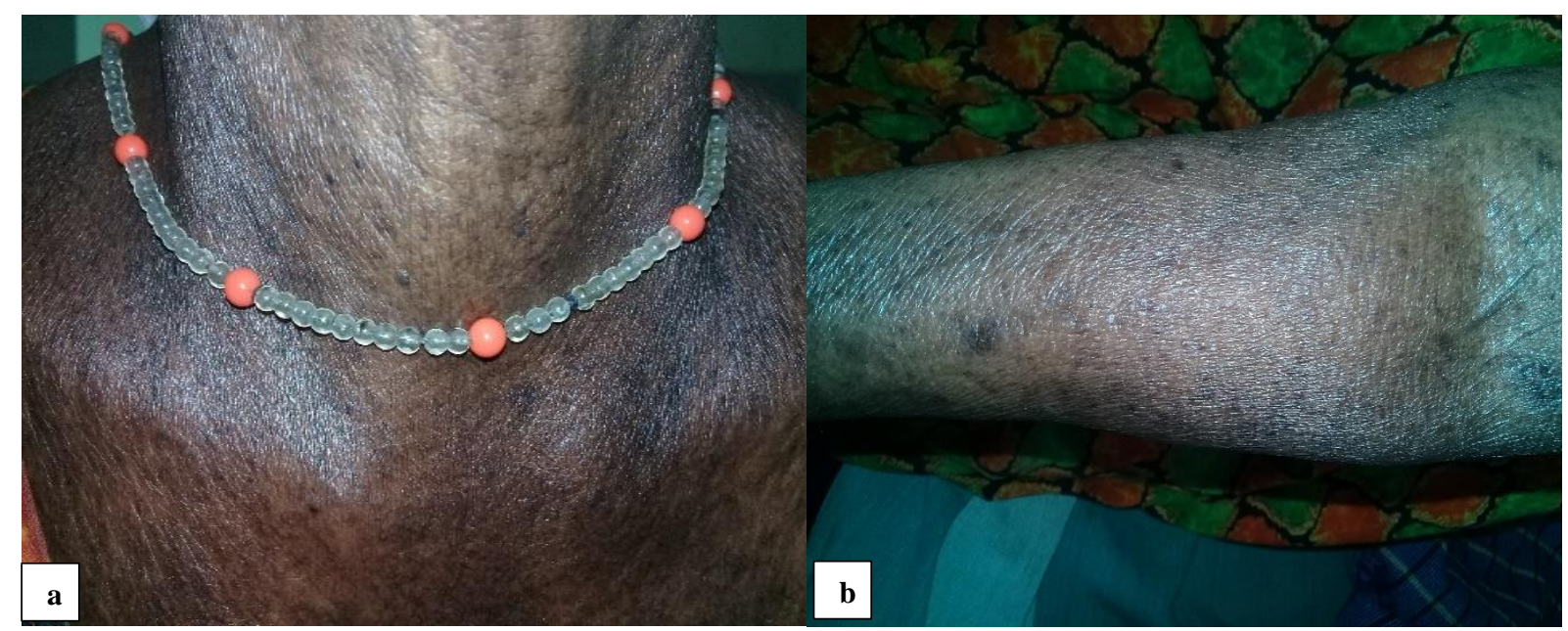

Figure 1: Patient with resolution of exfoliative lesions with residual hyperpigmentation 4 weeks after discontinuation of rifampicin.

Table 1: Assessment of causality, severity and preventability of adverse drug reactions.

\begin{tabular}{|llll|}
\hline Assessments & Case 1 & Case 2 & Case 3 \\
\hline Roussel Uclaf causality assessment & & Highly probable & Probable \\
\hline WHO-UMC system & Certain & Certain & Certain \\
\hline Naranjo algorithm causality assessment & Probable & Probable & Probable \\
\hline Modified Hartwig and Seigel severity scale & Moderate & Moderate & Moderate \\
\hline Schumock and Thornton preventability scale & Not preventable & Not preventable & Not preventable \\
\hline
\end{tabular}

Adenosine deaminase was $65 \mathrm{U} / \mathrm{l}(>40 \mathrm{U} / \mathrm{l})$ cytology revealed lymphocytic effusion (100\% lymphocytes).

Patient condition was improved and laboratory parameters were improved after one month. ATT drugs were reintroduced according to WHO recommendations. On rechallenge patient's liver parameters were elevated when started on isoniazid. Isoniazid was withdrawn. The therapy was modified and started with rifampicin, ethambutol, streptomycin, levofloxacin showed improvement.

\section{DISCUSSION}

Exfoliative dermatitis or erythroderma which is a severe form of cutaneous adverse drug reactions (CADR) described by Von Hebra in 1868. It was an inflammatory disorder and an extreme state of dysmetabolism characterized by extensive erythema and scaling all over the body classically involving more than $90 \%$ of the body surface area. ${ }^{12}$

Various causes of erythroderma in adults including preexisting eczema, psoriasis, lymphoma, leukemia and drugs such as phenylbutazone, hydantoin derivatives, carbamazepine, sulfonamides, penicillins, cimetidine, diltiazem, dapsone, allopurinol, gold salts, lithium and ATT drugs. Exposure to the causative drug may last for 2 weeks to several months before the reaction emerged. Drug-induced erythroderma had the best prognosis among all the causes of erythroderma often resolving in 2-6 weeks. ${ }^{13}$ Management usually involved withdrawal of offending drug and symptomatic treatment.

The underlying pathogenesis of this hypersensitivity, whether immune-mediated and/or toxic in nature, was unclear. Predisposing factors for hypersensitivity reactions to ATT included HIV infection, polypharmacy, advanced age, autoimmune disease and renal or liver impairment. ${ }^{14}$ In a large tertiary care center study on CADR with antitubercular drugs, pyrazinamide was the most common offending drug (2.38\%), followed by streptomycin $(1.45 \%)$, ethambutol $(1.44 \%)$, rifampicin $(1.23 \%)$ and isoniazid $(0.98 \%) .{ }^{15}$ Here, pyrazinamide, ethambutol and isoniazid were well-tolerated by our patient; however, she developed reaction to rifampicin.

Rifampicin generally was well tolerated. When given in usual doses, fewer than $4 \%$ patients with TB developed skin rashes $(0.8 \%) .{ }^{16}$ They usually commenced after four 
to eight weeks of therapy and must be recognition early to reduced associated morbidity and mortality.

In this case, the patient developed skin rashes after starting ATT and she improved after the withdrawal of ATT. She developed rashes again after rechallenge with rifampicin. So in our case she was started on modified regimen of isoniazid, ethambutol, levofloxacin and she tolerated well.

DILI is an uncommon cause of liver disease in the general population. In most cases was detected with mild to moderate elevations of liver tests. However, in rare cases it may lead to liver failure and mortality. ${ }^{17}$ Overall mortality from 10 to $17.3 \%$ had been observed in several series.

The major etiologic agents for DILI included antibiotics, anti-epileptics and non-steroidal anti-inflammatory drugs (NSAIDS). In India, TB remained a very common infectious disease, most commonly implicated drugs for DILI were ATT. Among the first-line drugs (isoniazid, rifampicin, pyrazinamide and ethambutol), the first three have the potential for hepatotoxicity with pyrazinamide being the most hepatotoxic followed by isoniazid and rifampicin. ${ }^{17}$

Pyrazinamide was associated with transient and asymptomatic elevations in serum aminotransferase levels and was a well-known cause of clinically apparent, acute liver injury that can be severe and even fatal. The onset of injury due to pyrazinamide was generally after 4 to 8 weeks and occasionally became apparent only after the pyrazinamide was stopped. The mechanism of hepatic injury by pyrazinamide was not known, may be caused by a metabolic intermediate. ${ }^{18}$

In case 2 , the patient presented with jaundice after initiation of first line ATT drugs. Liver parameters were markedly elevated. After discontinuation of ATT, the lab parameters and patient's clinical condition were improved. The liver parameters were elevated when he rechallenged with pyrazinamide. Then he started on streptomycin, levofloxacin, ethambutol and he well tolerated.

Despite the beneficial effects of isoniazid, hepatotoxicity was associated with INH therapies. About $18-20 \%$ of patients consuming INH had a transient elevation of serum alanine aminotransferase (ALT) level. ${ }^{19}$ Most of the patients can adapt to it and their serum ALT levels returned to normal without discontinuation, while some patients developed severe liver injury and even liver failure. Even though INH-induced liver injury had been known and extensively studied, its underlying mechanisms were still poorly understood.

In case 3 , the patient developed with jaundice after 10 days of ATT. All the liver function parameters were elevated. After discontinuation of ATT, liver parameters and clinical condition were improved. His liver parameters were elevated when isoniazid was reintroduced. Hence he started on modified therapy with rifampicin, pyrazinamide, streptomycin and ethambutol.

\section{CONCLUSION}

Here we reported one case of exfoliative dermatitis and two cases of drug induced liver injury due to first line ATT drugs. Finding the main cause and ruling out the definite drug to induce ADR is mainstay in preventing and managing any drug related diseases.

\section{ACKNOWLEDGEMENTS}

We want to thank our professor Dr. Davis Paul, head of the department, pulmonology for his guidance and support.

Funding: No funding sources

Conflict of interest: None declared

Ethical approval: Not required

\section{REFERENCES}

1. WHO Global tuberculosis report 2018. Geneva: World Health Organisation, 2018. Available at: https://apps.who.int/iris/handle/10665/274453.

2. WHO. Global tuberculosis control: surveillance, planning and financing. Geneva: World Health Organisation, 2005. Available at: https://apps.who.int/iris/handle/10665/144569

3. Michael OS, Sogaolu OM, Fehintola FA, Ige OM, Falade CO. Adverse events to first line antituberculosis drugs in patients co-infected with HIV and tuberculosis. Ann Ib Postgrad Med. 2016;14(1):21-9.

4. Castro AT, Mendes M, Freitas S, Roxo PC. Incidence and risk factors of major toxicity associated to first line antituberculosis drugs for latent and active tuberculosis during a period of 10 years. Rev Port Pneumol (2006). 2015;21(3):144-50.

5. Lv X, Tang S, Xia Y, Wang X, Yuan Y, Hu D, et al. Adverse reactions due to directly observed treatment strategy therapy in Chinese tuberculosis patients: a prospective study. PLoS One. 2013;8(6):65037.

6. Zhang Y, Wu S, Xia Y, Wang N, Zhou L, Wang J, et al. Adverse events associated with treatment of multidrug-resistant tuberculosis in china: an ambispective COHORT study. Med Sci Monit. 2017;23:2348-56.

7. Schnippel K, Berhanu RH, Black A, Firnhaber C, Maitisa N, Evans D, et al. Severe adverse events during second line tuberculosis treatment in the context of high HIV Co-infection in South Africa: a retrospective cohort study. BMC Infect Dis. 2016;16(1):593.

8. Tiberi S, Buchanan R, Caminero JA, Centis R, Arbex MA, Salazar M, et al. The challenge of the new tuberculosis drugs. Presse Med. 2017;46:41-51.

9. Vasava MS, Bhoi MN, Rathwa SK, Borad MA, Nair SG, Patel HD. Drug development against tuberculosis: 
Past, present and future. Indian $\mathrm{J}$ Tuberc. 2017;64(4):252-75.

10. Cardoso MA, Brasil P, Schmaltz CAS, Sant'Anna FM, Rolla VC. Tuberculosis treatment outcomes and factors associated with each of them in a COHORT followed up between 2010 and 2014. Biomed Res Int. 2017;2017:3974651.

11. Naik BR, Shastri SG, Vishwanath NS, Suryakanth MD, Sigarajipur A, Nair SA. Adverse drug rections in tuberculosis patients: consequences for TB control. Int J Tuberc Lung Dis. 2015;19(5):621-2.

12. Okoduwa C, Lambert WC, Schwartz RA, Kubeyinje E, Eitokpah A, Sinha S, et al. Erythroderma: review of a potentially life threatning dermatosis. Indian $\mathbf{J}$ Dermatol. 2009;54(1):1-6.

13. Breathnach SM. Drug reactions. In: Burns $T$, Breathnach S, Cox N, GriffithsC, eds. Rook's Textbook of Dermatology. 8th ed. United Kingdom: Wiley-Black well; 2010: 46-7.

14. Bharathiya PR, Joshi PB. Study of exfoliative dermatitis. Indian J Dermatol Venereol Leprol. 1995;61(2):81-3.
15. Tan WC, Ong CK, Kang SC, Razak MA. Two years review of cutaneous adverse reaction from first line anti-tuberculous drugs. Med J Malaysia. 2007;62(2):143-6.

16. Grosset J, Leventis S. Adverse effects of rifampicin. Rev Infect Dis. 1983;5(3):440-50.

17. Devarbhavi H. Antituberculous drug-induced liver injury: current perspective. Tropic Gastroenterol. 2011;32(3):167-74.

18. LiverTox: Clinical and Research Information on Drug-Induced Liver Injury. Bethesda (MD). National institute of diabetes and digestive and kidney diseases. 2012.

19. Sivannan S, Vishnuvardhan A, Muthu R. Isoniazidinduced liver disorder in the treatment of tuberculosis. Chronic Dis Transl Med. 201;4(4):268-70.

Cite this article as: Kolanchinathan E, Harichandran DT, Balakrishnan SM, Balakumaran SK. Adverse drug reactions to first line anti-tuberculosis drugs in newly diagnosed tuberculosis patients. Int J Basic Clin Pharmacol 2022;11:65-9. 